\title{
Attention deficit hyperactivity disorder secondary to lesion of the basal ganglia
}

\author{
Giovanni Mazzotta, Lucia Cirulli, Marco Sposato, Domenico Serino
}

Unit of Childhood Neuropsychiatry ASL04, Terni, Italy

Email: mazzogio@unipg.it

Received 27 September 2011; revised 7 November 2011; accepted 13 December 2011

\begin{abstract}
Attention Deficit Hyperactivity Disorder (ADHD) is a childhood onset autoregulation disorder characterized by attention deficit, hyperactivity and/or impulsiveness, which results in social and academic functional impairment. ADHD has a complex aetiology: along with genetic factors, anomalies in several cerebral districts have been reported. We describe the case of a 9-year old boy with glucose-6-phosphate dehydrogenase deficiency in association with memory, behavioural and attentive disabilities. The patient's clinical history is characterized by cerebral stroke at 3 years of age during a febrile episode, resulted in slight hemiparesis. Neuroimaging revealed a cystic lesion in the anterior portion of the right lenticular nucleus. Neuropsychological and psychiatric assessment evidenced alterations of executive functions. Diagnosis of ADHD secondary to lesion of the basal ganglia was made. We report the patient's clinical profile in the light of current evidence pointing towards dysfunction of the basal ganglia as a crucial aetiological factor in memory and executive function impairment.
\end{abstract}

Keywords: ADHD; Basal Ganglia; Attention Deficit; Memory Deficit; Stroke

\section{INTRODUCTION}

The Diagnostic and Statistical Manual for Mental Disorders (DSM-IV-TR) defines ADHD as an autoregulation disorder with onset before 6 years of age, characterized by the following symptoms: attentional deficits (low levels of sustained attention, poor ability in inhibiting distracting stimuli), hyperactivity (poor motor inhibition, avoidance of sedentary activities), and impulsiveness (rash behaviour, poor pulsional inhibition) [1].

Current scientific evidence suggests a neurobiological origin of the disorder related in particular to functional anomalies of two specific brain regions, the orbitofrontal cortex and the basal ganglia [2], which would result in abnormal responses to environmental stimuli. Ischemic stroke is rare in the paediatric age, and related reports in literature regarding postictal attentional and behavioural alterations are even rarer. We describe the case of a 9 -year old boy affected by glucose-6-phosphate dehydrogenase deficiency and a clinical history of cerebral stroke resulting in damage of the basal ganglia, with memory, behavioural and attentive disabilities.

\section{CLINICAL HISTORY}

The patient, affected by glucose-6-phosphate dehydrogenase (G6PD) deficiency, was sent to our institute for behavioural and attentional difficulties. His clinical history was characterized by cerebral stroke at 36 months of age during a febrile episode. The patient's parents reported the appearance of high levels of hyperactivity and impulsiveness following the ictal event, which became even more evident when the patient began attending school, due to difficulties in focusing on school work and maintaining adequate attention levels both in school and at home.

\section{CLINICAL EVALUATION}

\subsection{Neurological Examination}

Neurological assessment showed hyposthenia of the left inferior limb at the Mingazzini manoeuvre, asymmetry of osteotendinous reflexes with left hypoexcitability, dysmetria of the left superior limb with adiadochokinesis, athetosic movements of the fingers of the left hand when extended, spastic gait pattern.

\subsection{Neuroimaging}

MRI scan of the brain showed a cystic lesion in correspondence with the anterior portion of the right lenticular nucleus (see Figure 1).

\subsection{Neuropsychological Evaluation}

Cognitive level was determined through the administra- 


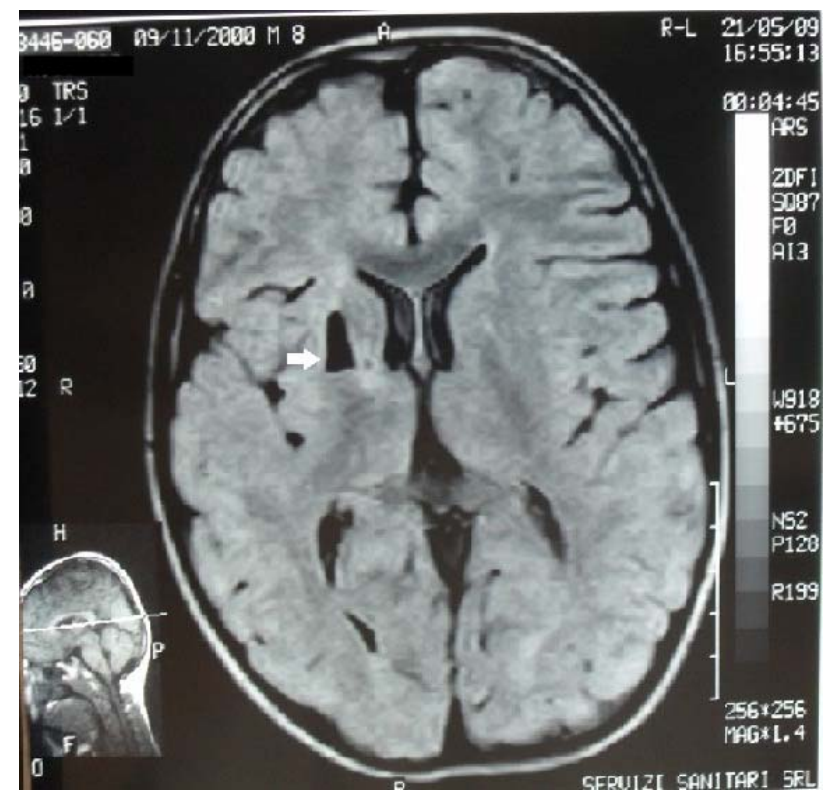

Figure 1. In correspondence with the anterior portion of the right lenticular nucleus, lesion of isointensity with liquor, surrounded by a thin hyperintense rim in PD and FLAIR, not causing mass-effect.

tion of the Wechsler Intelligence Scale for ChidrenThird Edition (WISC-III), from which the following scores were obtained: Verbal I.Q. 105, Performance I.Q. 85, Total I.Q. 95. Different tests were used to assess the patient's attentive, mnesic and learning abilities. The Childhood Memory and Learning Abilities Battery (PRO-MEA) [4] profile analysis, showed pathological scores in subtests concerning both short term memory and explicit long term memory, with the latter being inferior than the first, while long term implicit memory resulted normal. The Bells Test-Revised [5] showed values inferior to the 10th percentile for speed and accuracy, indicative of attentional pitfalls. Administration of the Italian Battery for $A D H D$ [6] resulted in values inferior to the 10th per-centile in the Frog Subtest (instrument for the evaluation of selective and sustained memory and motor inhibition) and in the Number Stroop Subtest (instrument for the evaluation of impulsiveness).

\subsection{Psychiatric Assessment}

Psychiatric assessment was undertaken through administration of semi-structured interviews and psychiatric questionnaires. The DSM-IV oriented Kiddie-Schedule for Affective Disorders and Schizophrenia-Present and Lifetime Version (K-SADS-PL) [7] semi-structured interview and the Scale for the Evaluation of Inattentive and Hyperactive Behaviour for the Child (SDAB-Cornoldi et al. 1996) were administered to the patient: both diagnostic instruments evidenced the presence of symptoms suggestive of ADHD. The K-SADS-PL interview, the
Scale for the Evaluation of Inattentive and Hy peractive Behaviour for Parents (SDAG-Cornoldi et al. 1994) and the Child Behaviour Check List for ages 6 - 18 (CBCL) [8] were administered to the patient's parents and were positive for ADHD. The Scale for the Evaluation of Inattentive and Hyperactive Behaviour for Teachers (SDAI-Cornoldi et al. 1994) was delivered to the teaching staff and was also positive for ADHD.

\section{DISCUSSIONS}

Clinical evaluation of our patient revealed the presence of ADHD. We know that ADHD is related to a dysfunction of the fronto-striatal network which includes the lateral prefrontal cortex (PCF), the anterior dorsal cingulate gyrus, the caudate nucleus, the putamen and the cerebellum. In particular, the PCF has a key role in learning and in executive functions, as in behavioural strategy programming; its hypofunctionality would entail the alteration of such functions. This circuitry receives stimuli from different associative areas which project towards the dorso-lateral PCF. In turn, the latter projects towards the basal ganglia and consequently to the thalamic nuclei which provide a feedback for the regulation of behaviour and problem-solving strategies [2]. In our patient, MRI scans revealed a cystic lesion of the right basal ganglia, precisely in the putamen and the caudate nucleus, entailing an alteration of the fronto-striatal circuitry assigned to the anterior attentional system. The lesion was secondary to the stroke triggered by the haemolytic process which followed a febrile episode: during haemolysis, hemoglobin binds with nitric oxyde (NO) determining its reduction. Depletion of NO results in vascular constriction and platelet aggregation, causing intravascular thrombosis and consequential stroke $[9,10]$.

Neuropsychological assessment revealed a dysfunction in executive functions. The patient had a poor performance in tasks involving sustained attention, problemsolving and regulation of inhibition. Both semantic memory and explicit long term memory were compromised, with pitfalls in logical memory. The resulting neuropsychological profile, in relation to the underlying anatomical lesion, can be superimposed to what is reported in literature regarding ADHD not associated with evident morphological alterations [11].

In conclusion, we believe that data obtained from the present case represents further evidence of the close relationship between ADHD and dysfunctionality of the executive function network [1-11]. Such close relation is not often easisly highlighted, given the low incidence of ischemic stroke during childhood and the rare occurrence of such a direct time-related appearance of ADHD symptomatology after a lesion of one of the cerebral structures 
involved in the executive function network [2].

\section{REFERENCES}

[1] Barkley, R.A. (1997) Behavioral inhibition, sustained attention and executive functions. Constructing a unifying theory of ADHD. Psychol Bull, 121, 65-94.

doi:10.1037/0033-2909.121.1.65

[2] Tripp, G. and Wickens, J.R. (2009) Neurobiology of ADHD. Neuropharmacology, 57, 579-589. doi:10.1016/j.neuropharm.2009.07.026

[3] Adams, H.P., Butler, M.J., Biller, J. and Toffol, G.J. (1986) Non hemorragic cerebral infarction in young adults. Archives of Neurology, 43, 793-796. doi:10.1001/archneur.1986.00520080041017

[4] Vicari, S. (2007) Promea, prove di memoria e apprendimento per l'età evolutiva. Giunti OS Organizzazioni Speciali, Florence.

[5] Biancardi, S. (1997) Test delle campanelle modificato. Psichiatria dell'infanzia e dell'adolescenza, 64, 73-84.
[6] Marzocchi, G.M., Re, A. and Cornoldi, C. (2010) Batteria italiana per l'ADHD per la valutazione dei bambini con deficit di attenzione/iperattività. Edizioni Erickson, Trento.

[7] Kaufman, J., Birmaher, B., Brent, D., Rao, U. and Ryan, N. (2004) K-SADS-PL Intervista diagnostica per la valutazione dei disturbi psicopatologici in bambini ed adolescenti. Edizioni Erickson, Trento.

[8] Achenbach, T.M. and Rescorla, L.A. (2001) Manual for the ASEBA school-age forms and profiles. University of Vermont, Burlington.

[9] Rother, R.P. and Bell, L. et al. (2005) The clinical sequelae of intravascular hemolysis and extracellular plasma hemoglobin. The Journal of the American Medical Association, 293, 1653-1662.

[10] Gladwin, M.T. and Kato, G.J. (2008) Hemosis-associated hypercoagulability in sickle cell disease: The plot (and blood) thickens. Haematologica, 93, 1-3. doi:10.3324/haematol.12318

[11] Emond, V., Joyal, C. and Poissant, H. (2009) Structural and functional neuranatomy of attention-deficit hyperactivity disorder. Encephale, 35, 107-114. 